\title{
Nonlinear Klein-Gordon equation pulsons with a fractional power potential
}

\author{
R.K. Salimov, E.G. Ekomasov ${ }^{\dagger}$ \\ †EkomasovEG@gmail.com
}

Bashkir State University, Zaki Validi 32, 450076, Ufa, Russia

The study of three-dimensional localized solutions of the nonlinear Klein-Gordon equation was conducted. The article describes new features of these solutions that distinguish them from other solutions. The consequences of such properties, which can be experimentally verified, were studied. The case where the Klein-Gordon equation is non-linear while solution amplitude tends to zero leads to the localization of solutions in a spherically symmetric case. The breather-like spherically symmetric solutions show the constancy of the frequency of the fast oscillation mode and are the three-dimensional objects. Such Lorentz-invariant breather-like solutions while moving are spatially modulated in the direction of motion similar to the de Broglie wave. For these solutions we a soliton interference pattern was considered that complements conventional Young's corpuscular and wave interference patterns on two slits. The experiment scheme to test such soliton interference pattern was created. Since such oscillating patterns tend to be localized, as a result of collision of such two objects, they can merge in one object. Due to the spatial modulation of the colliding objects, the motion direction of the resulting object will depend on the directions of the colliding objects, on their phase and oscillation frequency. When taking into account the localization of such oscillating objects, the interference pattern will be significantly different from the linear extended waves interference pattern. The characteristic feature of soliton interference is in the disappearance of the interference pattern on the screen when the distance between the slits is bigger than the value determined by the characteristic dimensions of the soliton.

Keywords: nonlinear Klein-Gordon equation, pulson.

\section{Introduction}

Nonlinear wave equations, for example, Klein-Gordon nonlinear differential equation, are very important for many areas of physics, including hydrodynamics, condensed matter physics, field theory, etc $[1,2]$. The most studied are $(1+1)$ - and the $(2+1)$-dimensional models [2-4]. These equations can be easily generalized for higher-dimensional spaces, for example, in the case of the spherical symmetry one has:

$$
u_{r r}+2 \frac{u_{r}}{r}-u_{t t}=F(u) .
$$

Stable solutions of these equations could be interpreted as the classical models of finite size particles. From this point of view, three-dimensional space-time equations are most interesting. The case where the Klein-Gordon equation is non-linear, for example, of the form

$$
u_{x x}+u_{y y}+u_{z z}-u_{t t}=u^{m / n}
$$

while solution amplitude tends to zero, leads to the solutions localization in a spherically symmetric case.

These solutions for confining models were considered, for instance, in $[5,6]$. These solutions represent non-spreading three-dimensional oscillating solutions, refer to the Fig. 1. The article describes new features of these solutions that distinguish them from other solutions. The consequences of such properties, which can be experimentally verified, were studied.

\section{On the possible interference of oscillating localized solutions}

The breather-like spherically symmetric solutions, for example, equations (2) show the constancy of the oscillations fast mode frequency and are three-dimensional objects. The presence of these properties opens up new possibilities in the interaction of such objects, for instance when they collide at different angles and while considering wave interference phenomenon. Such Lorentz-invariance breather-like solutions will undergo spatial modulation while moving. Indeed, let us suppose that there is some localized threedimensional breather-like solution with a preserved rapid fluctuations frequency. Then the solution

$$
u\left(r, t^{\prime}\right)=\sum_{i=1}^{N} a_{i}\left(r, t^{\prime}\right) \cos \left(\omega_{i} t^{\prime}\right)
$$

will be associated with some harmonic function $\cos \left(\omega_{j}\right.$ $t^{\prime}$ ), where $\omega_{j}$ - the rapid oscillations mode frequency and coefficient $a_{j}$ is substantially bigger than others.

In Lorentz transformation:

$x^{\prime}=\frac{x-v t}{\sqrt{1-v^{2} / c^{2}}}, \quad t^{\prime}=\frac{t-v x / c^{2}}{\sqrt{1-v^{2} / c^{2}}}, \quad y^{\prime}=y, \quad z^{\prime}=z$.

The function $\cos \left(\omega t^{\prime}\right)$ acquire the form

$$
\cos \left(\frac{\omega\left(t-v x / c^{2}\right)}{\sqrt{1-v^{2} / c^{2}}}\right)
$$


and the oscillating solution will be spatially modulated in the movement as a de Broglie wave [7]. An example of such transformation is a moving breather in the sine-Gordon equation of the form [1,2]:

$$
u=4 \operatorname{arctg}\left(\frac{\sin \left(a(t-v x) / \sqrt{1-v^{2}}\right)}{\operatorname{ch}\left(b(x-v t) / \sqrt{1-v^{2}}\right)}\right)
$$

which at motion with the speed $v$ spatially modulates as $\frac{a v}{\sqrt{1-v^{2}}}$, like de Broglie wave $\frac{m_{0} v h}{\sqrt{1-v^{2} / c^{2}}}$.

Spatial modulation of such constantly localized threedimensional solutions should manifest itself in waves interference phenomena - interference. This can be a very interesting situation of interference, similar to the hydrodynamic objects interference - the so-called walkers in Coude and Fort experiments [8]. Since such oscillating generations tend to be localized, at collision of such two objects, they can also be localized in one state. Indeed, let's consider, for example, the equation

$$
u_{r r}+2 \frac{u_{r}}{r}-u_{t t}=u^{m / n},
$$

where $m, n-$ are odd positive integers, while $m<n$. For $v=u r$, we obtain:

$$
v_{r r}-v_{t t}=r^{(n-m) / n} v^{m / n} .
$$

This equation shows that the field of the form $\mathrm{r}^{(\mathrm{n}-\mathrm{m}) / \mathrm{n}}$ will prevent spreading of the localized solution. It is natural to assume that at the collision of such two solutions they will localize in one state. Due to the spatial modulation of the colliding states, the motion direction of the resulting state will depend on the directions of the colliding states, on their phase and frequency oscillations. When taking into account the localization of such oscillating states, the interference pattern will be significantly different from the unlimited waves linear interference pattern.

Let's consider a simple modification of the Jung experiment scheme clearly separating these two cases. Fig. 2 shows such a scheme. The localized solutions pass through the diaphragm with two slits and come on the screen. $L-$ is the distance from the diaphragm with two slits to the screen, $d$ - is the distance between the slits, $A-$ is the characteristic dimensions of soliton-like state (SS) . Here, using the term «soliton-like», we mean only permanent localization of oscillatory states, and not their properties during collisions. While spreading at two slits initial localized state (or SS1), passing through two holes, splits into two localized SS2 and SS3. Then, SS2 and SS3 gather again in SS4, similar to SS1. SS4 directions of motion distribution can produce an interference pattern. Wave pattern of interference is provided by interaction of SS2 and SS3. It is natural to assume that the proportional increase in size of $L, d$ at $d>>A$, any interference pattern in «soliton» model must disappear.

Indeed, when SS1 is localized, it may pass simultaneously through two holes, only if the distance between them is not much bigger than its dimensions. In the case of conventional non-localized waves interference, where:

$$
\lambda / d=\Delta x / L
$$

$L, d$ proportional increase in size should not lead to a change in the interference pattern, while maintaining the coherence length and width. Thus, the cases of normal and soliton interference can be clearly divided. We emphasize once again that the soliton type of interference is possible, according to the authors, only for solutions which have the property of localization, such as equations solutions of the form (7). The numerical experiments to verify this model is quite time-consuming, as it is associated with three-dimensional calculations. Soliton interference model may also manifest itself in some real phenomena. Although soliton model particles are unorthodox hypotheses, as a real experiment to verify the soliton interference patterns one can offer a Tonomura experiment on electrons interference with a very large diameter of obstacles. Assuming endless length of wave coherence probability amplitude, conducting such an experiment seems superfluous. However, in reality, as we know, the double-slit experiment with such parameters has not been carried out.

\section{Conclusions}

In conclusion, we note that although the issue of particles viewed as solitons is not enough studied now and is very controversial, the soliton type of interference may occur in any events. The characteristic feature of soliton interference is in the disappearance of the interference pattern on the screen when the distance between the slits is larger than the value determined by the characteristic dimensions of the soliton. In conventional interference the proportional increase of $L, d$ parameters does not affect it. Therefore, the proposed experiment scheme to verify the existence of such interference deserves, in the authors opinion, further investigation.

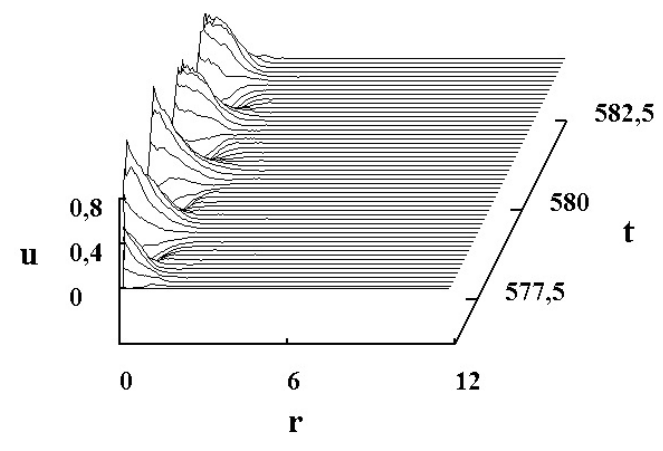

Fig. 1. Numerical solution of the equation (2) for $m=3, n=7$.

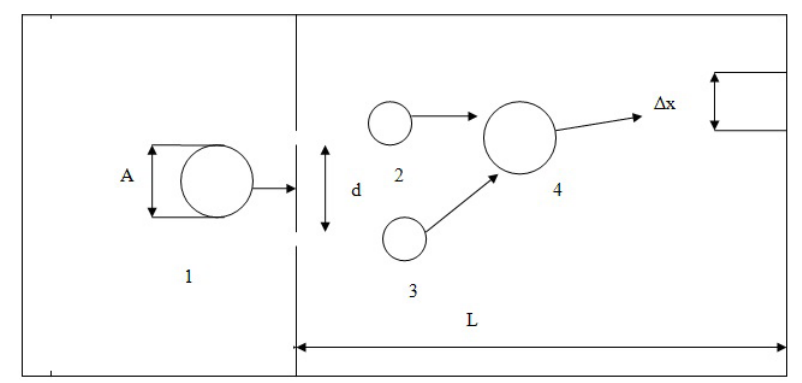

Fig. 2. Two-slit experiment scheme to test the soliton interference pattern. 


\section{References}

1. T. Dauxois, M. Peyrard, Physics of Solitons, N.Y. Cambridge University Press, (2010).

2. O. M. Braun, Y.S. Kivshar. The Frenkel-Kontorova Model. Concepts, Methods, and Applications. Springer (2004).

3. J. A. Gonzalez, A. Bellorin, L. E. Guerrero, Solitons and Fractals. 33, 143 (2007).

4. D. Saadatmand. J. Kurosh, Braz. J. Phys. 56, 43 (2013).
5. E. G. Ekomasov, R. K. Salimov, JETP Lett. 100 (7) 532. (2014),

6. E. G. Ekomasov, R. K. Salimov, JETP Lett. 102(2),122 (2015).

7. R.P. Feynman, R.B. Leighton, M. Sands. The Feynman Lectures on Physics. Vol. 3. Quantum Mechanics, Adidson-Wesley publishing company (1963) 312 p.

8. Y. Couder, E. Fort, Phys. Rev. Lett. 97, 154101 (2006). 